\title{
28 Research Suare \\ A Preliminary Study of Personalized Head CT Examination in Pediatric
}

\section{BingYang Bian}

Jilin University First Hospital

\section{BoXu Zhou}

first hospital of jilin university

\section{Gang Wang}

China-Japan Union Hospital of Jilin University

\section{QingChen Zhou}

Jilin University First Hospital

\section{ShuJia Xu}

Jilin University First Hospital

Dan Li ( 13604430730@126.com )

Jilin University First Hospital

\section{Research article}

Keywords: head CT, head circumference, tube current, sensitive organs, brain, eye lens, salivary glands

Posted Date: December 24th, 2019

DOl: https://doi.org/10.21203/rs.2.19510/v1

License: (c) (1) This work is licensed under a Creative Commons Attribution 4.0 International License. Read Full License 


\section{Abstract}

Background: Same head CT examination protocol was employed for pediatric patients who's skull sizes are different, this could be excessive radiation doses because they usually have smaller head circumference. In our study, we investigate if $\mathrm{mAs}$ according to head circumference $(\mathrm{HC})$ reduce radiation doses of sensitive organs including brain, eye lens and salivary glands, but could keep the image quality.

Methods: 83 pediatric patients were prospectively selected. Without limiting the head circumference, 15 pediatrics were selected as conventional group by random number method and received routine head CT examination protocol (250mAs). Low-dose group including remaining 68 patients were divided into 3 subgroups based on HC: $54.1-57.0 \mathrm{~cm}$ for group $A(200 \mathrm{mAs}), 51.1-54.0 \mathrm{~cm}$ for group B(150mAs), 48.1$51.0 \mathrm{~cm}$ for group $\mathrm{C}(100 \mathrm{mAs})$. The Image quality was assessed by subjective and objective image score. Radimetrics was used to calculate radiation doses of sensitive- organs.

Results: In the conventional group, pediatric patients with smaller head circumference receive higher radiation doses of sensitive-organs. Radiation doses of brain and salivary glands were negatively correlated with HC. The radiation dose of sensitive-organs in 3 low-dose subgroups were significantly lower than conventional group. The subjective image quality scores in group A and B was no statistical different than conventional group. The SNR of thalamus and centrum ovale in low-dose subgroups were no statistical differences compared with conventional group.

Conclusions: Our research indicates that personalized brain CT examination in pediatrics can reduce the radiation doses of sensitive-organs but keep image quality. $\mathrm{HC}$ can serve as an effective index to guide personalized head CT scan.

\section{Background}

Head CT scan has become a routine clinical examination in neurosurgery, neurology and emergency department. Although head CT is a relatively efficient diagnostic procedure as well as offers high diagnostic value, it is also a major contributor to collective radiation dose in daily diagnostic radiology practice [1 - 3]. Thus, the increasing use of brain CT scans raises the concern of a possible health impact of ionizing radiation exposure. Pediatrics are in growth stage, the speed and proportion of cell division and renewal are much faster than adults, therefore, they are more sensitive to $\mathrm{X}$-ray exposure $[4,5]$. Receiving the same radiation doses, pediatrics have a higher risk of radiation exposure injury than adults, meanwhile, a lifetime risk of cancer is much higher compared with adults [6-9]. Thus, reducing radiation doses from pediatrics' brain CT under optimal image quality has been a hot research topic [10,11]. Personalized brain CT examination protocol is required in clinical practice but an effective index factor to guide the brain CT protocol has not been established [12]. CTDI ${ }_{\text {vol }}$ is not a direct measurement or estimate of patient absorbed radiation doses. The size of patient may affects the absorbed dose: if all other factors remain the same, the smaller patient scanned with the same CTDI ${ }_{\text {vol }}$ will have a higher absorbed dose than a lager patient [13]. 
In this study, we use head circumference $(\mathrm{HC})$ as an index to determine the mAs of brain CT. The sensitive organs including brain, eye lens, salivary glands employed to evaluate the radiation exposure. Subjective and objective image quality score was used to evaluate the image quality.

\section{Methods}

\section{Participants}

This research was approved by the ethics committee of Jilin University (date of approval: February 1 th 2017) and written informed consent was provided to every participant. During a 7-month period from February to September 2017, pediatric patients were recommended to undergo a non-contrast head CT examination for various clinical symptoms form neurosurgery, neurology and emergency department. Finally, 83 patients are recruited in the study, male 47, female 36, age 1-17 years old, their demographic characteristics are summarized in Table1. 15 patients were selected as the conventional group by random number method, who receive standard brain CT protocol(tube current $250 \mathrm{mAs}$ ). The range of head circumference is $48.1-59.2 \mathrm{~cm}(53.75 \pm 3.14 \mathrm{~cm})$. The other 68 patients were randomly separated into 3 low-dose group based on the HC: group A, $54.1-57.0 \mathrm{~cm},(56.00 \pm 0.24 \mathrm{~cm})$; group B, $51.1-54.0 \mathrm{~cm}$, $(52.98 \pm 0.20 \mathrm{~cm})$; group $C, 48.1-51.0 \mathrm{~cm},(49.54 \pm 0.23 \mathrm{~cm})$. General information and scan parameters for conventional and low-dose group is in Table2.

\section{Scanning Protocol}

All subject's head CT examination were performed with a 64-slice MDCT scanner (Light Speed, GE Healthcare, USA) in the axial plane with the patient in the supine position. The tube current in conventional group was $250 \mathrm{mAs}$. The tube currents in low-dose groups were $200 \mathrm{mAs}, 150 \mathrm{mAs}$ and $100 \mathrm{mAs}$ for group $A, B$ and $C$, respectively. The following remaining scanning parameters were the same for four groups: $120 \mathrm{kVp}, 5 \mathrm{~mm}$ section thickness, $0.45 \mathrm{~mm}$ interval, $360 \mathrm{~ms}$ rotation time and a FOV of $38 \mathrm{~mm} \times 38 \mathrm{~mm}$.

\section{Organ-Specific-Radiation Dose Levels Estimation}

Radimetrics, researched and developed by Byer Healthcare from Germany, an analysis platform based on Monte-Carlo-Simulation was used to calculate the radiation doses of sensitive organs. ${ }^{14,15}$ The total organ dose is calculated first for each slice using the CTDI $\mathrm{vol}_{\text {lat }}$ at that slice and then summed over the slices that all into the scan region:

\section{See Formula 1 in the Supplemental Files}

Where coeff is the ratio of the simulated organ to the simulated CTDI $\mathrm{vol}_{\text {al }}$ as described above, and $i$ indicates slice specific values.

\section{Subjective image quality score assessment}


Subjective image quality analysis was performed by two neuroradiologists with 8 and 20 years' experience, respectively. The neuroradiologists were blind to the scanner, scan parameters and slice thickness . Images were displayed using a fixed brain window setting (window width, $80 \mathrm{Hu}$; window level, $40 \mathrm{Hu}$ ). In the subject image quality assessment (1) severity of background noise, (2) severity of imaging artefacts, and (3) clarity of demonstration of lesions and anatomical structure were taken into account. Five grade were designed to each factor with 5 representing the best quality (Table 3 ). The scores from the three domains determined by the two neuroradiologist were then averaged to generate an overall score for image quality. An image quality score $\geq 3$ was consider a qualified image for the demand of diagnostic.

\section{Objective image quality score assessment}

All head CT images measurement were carried out on GE workstation ADW4.4 in our department. The bilateral thalamus and centrum ovale were selected to place equal ROls by a 10 years' experience neuroradiologist who were blind to the scanner, scan parameters and slice thickness. Average values on both sides. The standard deviation (SD) of mean CT density (Hounsfield unit) was used to measure the noise. The signal to noise ratio (SNR) was the HU/SD . The representative image measurement in FIG 1.

\section{Statistical analysis}

All data were analyzed using SPSS (Version 22; IBM, New York, USA). An initial analysis was performed using the Anderson-Darling test to evaluate whether the data were normally distributed. Numerical data were expressed as means \pm standard deviation (SD) and compared by Mann-Whitney $U$ test or ANOVA, as appropriate. ANOVA test was used to compare the difference of radiation dose and image quality score of sensitive organs in each group. When the difference was significant, tukey-kramer pairwise comparison would be conducted. Wilcoxon rank sum test was used for non-normal distribution. The consistency of the analysis results of two radiologists was checked by Kappa-test. The $K$ value $>0.6$ was good, $0.4 \leq K$ value $\leq 0.6$ was moderate, and the $K$ value $<0.4$ was poor. The statistical significance level was set as $\mathrm{P}<0.05$.

\section{Results}

\section{The relationship between head circumference and radiation doses of sensitive organs}

In the convention group, we found that the radiation doses of sensitive-organs of pediatric patients with smaller $\mathrm{HC}$ increase under same $\mathrm{mAs}$. The radiation doses of brain fell a negative liner correlation with the $\mathrm{HC}\left(\mathrm{R}^{2}=0.565\right)$. F-test showed that the $\mathrm{HC}$ has a significant impact on the brain radiation doses $(P=0.00124)$. The radiation doses of salivary glands also showed a negative liner correlation with the $\mathrm{HC}$ $\left(R^{2}=0.268, P=0.048\right)$. On the other hand, the radiation doses of eye lens were not linearly correlated with the $\mathrm{HC}\left(\mathrm{R}^{2}=0.096, P=0.26\right)$, although the radiation dose seems decrease for larger $\mathrm{HC}$, as shown in FIG 2 .

\section{Radiation absorption benefit of low-dose head CT scan}


With the decrease of $\mathrm{mAs}$, the radiation doses of sensitive-organs also decreased. Compared with the convention group, the radiation doses of brain, eye lens and salivary glands in low-dose group decreased significantly ( $P \otimes 0.05)$, as shown in Table 4. When tube current have reduced to 200,150 and $100 \mathrm{mAs}$, the radiation doses respectively decreased $25 \% \varangle 33 \% \varangle 49 \%$ in brain $₫ 20 \% \varangle 37 \% \varangle 50 \%$ in eye lens $₫ 26 \% \varangle 34 \% \varangle 57 \%$ in salivary glands compared with $250 \mathrm{mAs}$.

\section{Subjective image quality in convention and low-dose head CT scan}

There was not significant disagreement between the two radiologists. The Kappa value of the analysis results of two radiologists was $\mathrm{K}=0.741$. The average subjective image quality score of convention and low-dose group were shown in Table 5. Subjective image quality score of $A(200 \mathrm{mAs})$ and B (150 mAs) group was lower than convention group, but there was no statistical difference $(P \otimes 0.05)$. Image quality score of $\mathrm{C}$ group (100 mAs) was significantly lower than convention group $(P<0.05)$. Although the mAs had been decreased, the image quality score didn't fell lower than 3 score and are acceptable for diagnose purpose.

\section{Objective image quality in convention and low-dose head CT scan}

With the increase of mAs in low-dose group, the SNR of thalamus and centrum ovale increased gradually. The mean CT density, SD and SNR of thalamus and centrum ovale in convention and low-dose group were shown in Table 6. In the evaluation of thalamus and centrum ovale, there were no significant differences in SD and SNR (Pख0.05).

\section{Discussion}

Head CT scan accounted for more than $50 \%$ of pediatric total CT examination $[11,16,17]$. The gene mutation and cancer risk related with X-radiation is 10 times higher than adults, especially, brain, eye lens and salivary glands are sensitive to radiation in head CT scan $[18,19]$. Pediatrics are in the stage of growth and development, even in the same age group, the $\mathrm{HC}$ will vary greatly, so age is not an indicator of the HC. It is unreasonable for pediatric patients at different age to use same mAs when performing head CT examination. Age is not a reasonable indicator for choosing mAs.

One of the most important reasons is that it is impossible/difficult to accurately estimate the radiation doses of each organ, so it is impossible to quantitatively evaluate the benefits of low-dose brain CT examination. $\mathrm{CTDI}_{\mathrm{vol}}$ is not an accurate representation of the dose absorbed by individual patients, and dose not provide dose to the organ or allow a comparison of dose across different modalities that deliver ionizing radiation.

We use Radimetrics software to evaluate radiation doses of sensitive-organs. Our found that brain radiation doses varied among pediatric patients of conventional group, this indicate that some patients might have received excessive radiation doses during head CT scan using $250 \mathrm{mAs}$. Pediatrics with smaller $\mathrm{HC}$ may receive overdose for brain, eye lens and salivary glands. One possible explanation for the 
negative correlation between brain radiation doses and $\mathrm{HC}$ is that the skull of larger circumference absorb more radiation than smaller ones. In same $\mathrm{mAs}$, a same amount of radiation doses was delivered to the subject, the larger skulls absorb more radiation doses and result a lower radiation doses for the brain. If this is true, the organ protected by the skull or craniofacial bones should show a similar correlation with $\mathrm{HC}$ and the organ outside of the skull might not fell a negative liner correlation with $\mathrm{HC}$. To test this, we exam the radiation dose in salivary glands (protected by mandible) and eye lens (not protected by bone), respectively. Our data show that there is a negative correlation between brain radiation doses and head circumference in the conventional group. Similarly, the salivary glands are protected by the mandible, and their radiation absorption is negatively correlated with the head diameter, similar to that of the brain. However, there was no significant linear relationship between radiation dose received by eye lens without skull or craniofacial bones protection and head circumference. It is essential that this results have provided theoretical support for the application of different mAs based on different $\mathrm{HC}$ in low-dose group.

We then investigate how the low dose head CT scan can benefit the pediatric patients. In our study, lowdose groups were divided into 3 subgroups according to HC,tube current were respectively $200 \mathrm{mAs}$, $150 \mathrm{mAs}, 100 \mathrm{mAs}$ for group A, B, C. Conventional group carried out fixed $250 \mathrm{mAs}$ not basing HC. We then test if we can use lower dose head CT scan in patients with small HC and still keep the image quality. Subjective image quality scores in low-dose and conventional group were greater than or equal to 3 scores to meet image diagnosis. There was no significant difference between the $250 \mathrm{mAs}$ and the $200 \mathrm{mAs}, 150 \mathrm{mAs}$ scans in subjective image quality score, but there was a significant difference in the image quality score between the $250 \mathrm{mAs}$ and the $100 \mathrm{mAs}$ scans. In the group Clthe image score of the four patient was 3 , which was the lowest acceptable quality of the clinical diagnosis, which indicated that quality of image under tube current below the $100 \mathrm{mAs}$ was unacceptable.

We found that personalized brain CT scan in low-dose group can significantly reduce the organ-specificradiation dose levels than conventional group but keep image quality. $\mathrm{HC}$ can be used as an indicator for selecting mAs in head CT examination. Personalized head CT scan can reduce the radiation dose efficiently. Therefore, it is more objective and appropriate to select mAs using this method during head CT scans in pediatric patients.

But, no specific rules are found to further refine the relationship between $\mathrm{HC}$ and tube current and radiation dose. As for the $\mathrm{HC}$ and the specific mAs value should be set, further study is needed.

\section{Conclusions}

Selection of $\mathrm{mAs}$ according to different $\mathrm{HC}$ can be more rational and personalized design, so our research indicates that $\mathrm{mAs}$ basing on $\mathrm{HC}$ at head $\mathrm{CT}$ examination can effectively reduce organ-specific-radiation doses without compromise of image quality. The HC could be an effective index factor to guide personalized head CT scan.

\section{Declarations}


Acknowledgements

Not applicable.

\section{Author's contribution}

BBY is in charge of result analysis and thesis writing; ZQC is in charge of image evaluation; $Z B X$ in charge of scanning; WG in charge of children's head circumference survey; XSJ is in charge of original data collection; LD is in charge of guiding scheme design and paper revision. All authors have read and approved the manuscript", and ensure that this is the case.

\section{Funding}

Jilin Province Science and Technology Development Plan Item (NO. 20191102001YY). Funder is the corresponding author of this article.

\section{Availability of data and materials}

The datasets generated and analysed during the current study are not publicly available due it involves the privacy of patients but are available from the corresponding author on reasonable request.

\section{Ethics approval and consent to participate}

Ethical approval was provided by the first hospital of Jilin University (Ethical approval reference number: 2017-359). Informed consent was written obtained when patients were admitted to hospital. Written informed consent was obtained from a parent or guardian for participants under 16 years old.

\section{Consent for publication}

Not applicable.

\section{Competing interests}

The authors declare that they have no competing interests.

\section{Abbreviations}

HC: head circumference; SD: standard deviation; SNR: signal to noise ratio; THL: thalamus; CO : centrum ovale

\section{References}

1. Sodickson A, Baeyens PF, Andriole KP, Prevedello LM, Nawfel RD, Hanson R, et al. Recurrent CT, cumulative radiation exposure, and associated radiation-induced cancer risks from CT of adults. Radiology. 2009; 251(1): 175-184. 
2. Costello JE, Cecava ND, Tucker JE, Bau JL. CT radiation dose: current controversies and dose reduction strategies. AJR Am J Roentgenol. 2013; 201(6): 1283-1290.

3. Coakley FV, Gould R, Yeh BM, Arenson RL. CT radiation dose: what can you do right now in your practice. AJR Am J Roentgenol. 2011; 196(3): 619-625.

4. Bertell R, Ehrle LH, Schmitz-Feuerhake I. Pediatric CT research elevates public health concerns: lowdose radiation issues are highly politicized. Int J Health Serv. 2007; 37(3): 419-439.

5. Kalra MK, Maher MM, Toth TL, Hamberg LM, Blake MA, Shepard JA, et al. Strategies for CT radiation dose optimization. Radiology. 2004(3); 230: 619-628.

6. Einstein AJ, Henzlova MJ, Rajagopalan S. Estimating risk of cancer associated with radiation exposure from 64-slice computed tomography coronary angiography. JAMA. 2007; 298(3): 317-323.

7. Brenner D, Elliston C, Hall E, Berdon W. Estimated risks of radiation-induced fatal cancer from pediatric CT. AJR Am J Roentgenol. 2001; 176(2): 289-296.

8. Golding SJ, Shrimpton PC. Commentary. Radiation dose in CT: are we meeting the challenge. Br J Radiol. 2002; 75(889): 1-4.

9. de González A B, Darby S. Risk of cancer from diagnostic X-rays: estimates for the UK and 14 other countries. Lancet. 2004; 363(9406): 345-351.

10. Weinman JP, Mirsky DM, Jensen AM. Dual energy head CT to maintain image quality while reducing dose in pediatric patients. Clin Imaging. 2019; 55: 83-88.

11. Ghotbi N, Ohtsuru A, Ogawa Y, Morishita M, Norimatsu N, Namba H, et al. Pediatric CT scan usage in Japan: results of a hospital survey. Radiat Med. 2006; 24(8): 560-567.

12. Hardy AJ, Bostani M, Hernandez AM, Zankl M, McCollough C, Cagnon C, et al. Estimating a sizespecific dose for helical head CT examinations using Monte Carlo simulation methods. Med Phys. 2019; 46(2): 902-912.

13. Hoye J, Sharma S, Zhang Y, Fu W, Ria F, Kapadia A, et al. Organ Doses from CT Localizer Radiographs: Development, Validation, and Application of a Monte Carlo Estimation Technique. Med Phys. 2019; 46(11):5262-5272.

14. Li X, Yang K, DeLorenzo MC, Liu B. Assessment of radiation dose from abdominal quantitative CT with short scan length. Br J Radiol. 2017; 90(1074): 20160931.

15. Weis M, Henzler T, Nance JW, Haubenreisser H, Meyer M, Sudarski S,bet al. Radiation Dose Comparison Between $70 \mathrm{kVp}$ and $100 \mathrm{kVp}$ With Spectral Beam Shaping for Non-Contrast-Enhanced Pediatric Chest Computed Tomography: A Prospective Randomized Controlled Study. Invest Radiol. 2017; 52(3): 155-162.

16. Verdun FR, Gutierrez D, Vader JP, Aroua A, Alamo-Maestre LT, Bochud F, et al. CT radiation dose in children: a survey to establish age-based diagnostic reference levels in Switzerland. Eur Radiol. 2008; 18(9): 1980-1986.

17. Bernier MO, Rehel JL, Brisse HJ, Wu-Zhou X, Caer-Lorho S, Jacob S, et al. Radiation exposure from CT in early childhood: a French large-scale multicentre study. Br J Radiol. 2012; 85(1009): 53-60. 
18. Karatasakis A, Brilakis HS, Danek BA, Karacsonyi J, Martinez-Parachini JR, Nguyen-Trong PJ.

Radiation-associated lens changes in the cardiac catheterization laboratory: Results from the ICCATARACT (CATaracts Attributed to RAdiation in the CaTh lab) study. Catheter Cardiovasc Interv. 2018; 91(4): 647-654.

19. Hall EJ. Lessons we have learned from our children: cancer risks from diagnostic radiology. Pediatr Radiol. 2002; 32(10): 700-706.

\section{Tables}

Table1. Demographic characteristics of subjects

\begin{tabular}{lc}
\hline Sex (male: female) & $47: 36$ \\
Mean Age (SD) & $8.5(4.7)$ \\
Symptoms* & \\
headache & 41 \\
dizzy & 21 \\
nausea & 26 \\
vomiting & 12 \\
trauma & 10 \\
\hline
\end{tabular}

*Multiple symptoms were reported by some patients

Table2. General information and scan parameters of convention and low-dose group

\begin{tabular}{|c|c|c|c|c|c|c|}
\hline \multirow[t]{2}{*}{ Group } & \multirow[t]{2}{*}{ Number } & \multicolumn{2}{|c|}{ Gender } & \multirow[t]{2}{*}{$\mathrm{HC}(\mathrm{cm})$} & \multirow[t]{2}{*}{ Age(years old) } & \multirow[t]{2}{*}{$\mathrm{mAs}$} \\
\hline & & M & $\mathrm{F}$ & & & \\
\hline Convention & 15 & 10 & 5 & $54.30 \pm 0.82$ & $10.60 \pm 1.33$ & 250 \\
\hline \multicolumn{7}{|l|}{ Low-dose } \\
\hline $\mathrm{A}$ & 22 & 11 & 11 & $56.00 \pm 0.24$ & $13.14 \pm 0.60$ & 200 \\
\hline B & 24 & 14 & 10 & $52.98 \pm 0.20$ & $8.17 \pm 0.65$ & 150 \\
\hline $\mathrm{C}$ & 22 & 12 & 10 & $49.54 \pm 0.23$ & $3.55 \pm 0.38$ & 100 \\
\hline
\end{tabular}


Table 3. Grading scale for subjective image quality

\begin{tabular}{llll}
\hline Reader Score & \multicolumn{1}{c}{ Noise } & \multicolumn{1}{c}{ Artefacts } & \multicolumn{1}{c}{ Anatomical details and lesion } \\
\hline 5 & no image noise & no artefacts & clearly \\
4 & minimum image noise & minimum & owed clear details and lesion \\
3 & less average noise & artefacts & owed clear details, lesion appeared well \\
& & artefacts are & identification of anatomical details was difficult, lesions \\
2 & above average noise & obvious & shown were not clear \\
& & but acceptable & unable to identify detail and lesions \\
& unacceptable image noise & artefacts & \\
& & affecting & \\
& diagnostic & \\
& information & \\
& & no applicable \\
&
\end{tabular}

Table 4. Comparison of radiation doses of sensitive organs of pediatrics with brain CT in convention and low-dose group

\begin{tabular}{|c|c|c|c|c|}
\hline Group & Number & Brain & Eye lens & Salivary glands \\
\hline Convention & 15 & $34.37 \pm 3.62$ & $41.54 \pm 1.04$ & $35.04 \pm 4.94$ \\
\hline \multicolumn{5}{|l|}{ Low-dose } \\
\hline $\mathrm{A}$ & 22 & $25.91 \pm 0.99 *$ & $33.03 \pm 0.35 *$ & $25.92 \pm 0.99 *$ \\
\hline B & 24 & $23.18 \pm 6.11 *$ & $26.18 \pm 2.72 *$ & $22.93 \pm 6.54^{*}$ \\
\hline $\mathrm{C}$ & 22 & $17.38 \pm 3.23 *$ & $20.88 \pm 4.45 *$ & $14.96 \pm 2.67 *$ \\
\hline
\end{tabular}

\footnotetext{
$*_{\square}$ Compare with convention group; All $P \llbracket 0.05$
} 
Table 5. Subjective image quality score of patient with head CT between convention and low-dose group

\begin{tabular}{|c|c|c|c|c|c|c|c|c|}
\hline \multirow[t]{2}{*}{ Group } & \multirow[t]{2}{*}{ Number } & \multicolumn{4}{|c|}{ Reader Score } & \multicolumn{2}{|c|}{ Mean Score } & \multirow[t]{2}{*}{$P$ value } \\
\hline & & 5 & 4 & 3 & 2 & 1 & & \\
\hline Convention & 15 & 15 & 0 & 0 & 0 & 0 & $5.00 \pm 0.00$ & प \\
\hline \multicolumn{9}{|l|}{ Low-dose } \\
\hline A & 22 & 20 & 2 & 0 & 0 & 0 & $4.90 \pm 0.49$ & $0.389 *$ \\
\hline B & 24 & 17 & 7 & 0 & 0 & 0 & $4.67 \pm 0.49$ & $0.097 *$ \\
\hline $\mathrm{C}$ & 22 & 7 & 11 & 4 & 0 & 0 & $4.13 \pm 0.61$ & $\square 0.001 *$ \\
\hline
\end{tabular}

* $\llbracket$ Compare with convention group

Table 6. Comparison of mean CT density, SD and SNR of thalamus and centrum ovale in convention and low-dose group

\begin{tabular}{|c|c|c|c|c|c|c|c|}
\hline \multirow[t]{2}{*}{ Group } & \multirow[t]{2}{*}{ Number } & \multicolumn{2}{|c|}{ CT density(HU) } & \multicolumn{2}{|c|}{$\mathrm{SD}$} & \multicolumn{2}{|c|}{ SNR } \\
\hline & & THAL & $\mathrm{CO}$ & THAL & $\mathrm{CO}$ & THAL & $\mathrm{CO}$ \\
\hline Convention & 15 & $37.61 \pm 3.50$ & $26.76 \pm 2.21$ & $2.09 \pm 0.42$ & $1.58 \pm 0.28$ & $18.68 \pm 3.56$ & $17.47 \pm 3.73$ \\
\hline \multicolumn{8}{|l|}{ Low-dose } \\
\hline A & 22 & $37.84 \pm 3.43$ & $26.87 \pm 2.20$ & $2.33 \pm 0.46$ & $1.98 \pm 0.29$ & $16.77 \pm 2.88$ & $15.55 \pm 2.82$ \\
\hline B & 24 & $37.47 \pm 3.67$ & $26.29 \pm 2.11$ & $2.58 \pm 0.51$ & $2.02 \pm 0.31$ & $15.06 \pm 2.81$ & $13.72 \pm 2.43$ \\
\hline $\mathrm{C}$ & 22 & $38.17 \pm 3.23$ & $26.97 \pm 2.15$ & $2.89 \pm 0.57$ & $2.34 \pm 0.37$ & $13.66 \pm 2.54$ & $11.78 \pm 2.19$ \\
\hline$P$ value & & 0.43 & 0.02 & 0.11 & 0.07 & 0.54 & 0.19 \\
\hline
\end{tabular}

Note: THL indicates thalamus; CO indicates centrum ovale

Figures

Figure 1 
ROI was selected in bilateral thalamus and centrum ovale

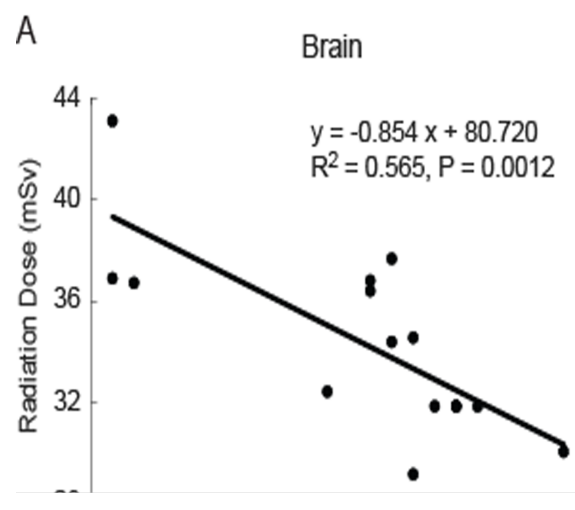

B

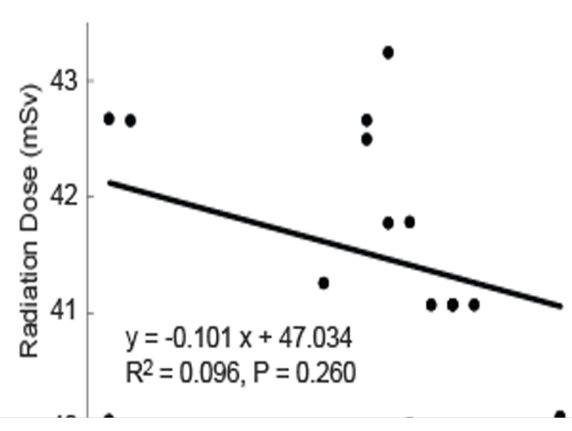

C

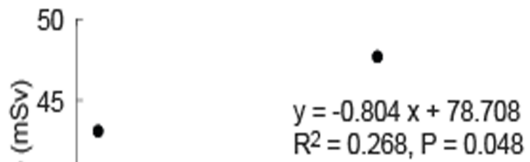

$\$ 40$

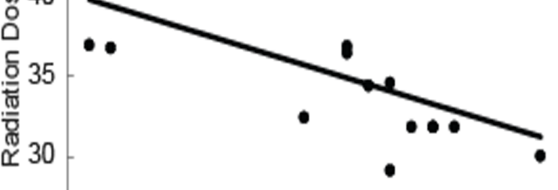

Figure 2

Relationship between head circumference and radiation doses of sensitive-organ in convention group. $A \rrbracket$ brain $₫ \mathrm{~B} \bigotimes$ eye lens $₫ \mathrm{C} \llbracket$ salivary glands

\section{Supplementary Files}

This is a list of supplementary files associated with this preprint. Click to download.

- formula1.JPG 